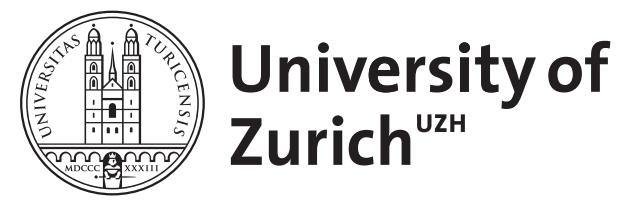

\title{
Differenzialdiagnose Nausea und Erbrechen
}

\author{
Janiak, P ; Fried, M
}

\begin{abstract}
Zusammenfassung: Nausea und Erbrechen sind häufig. Sie können eine physiologische Antwort auf ein exogenes Toxin sein, aber auch Ausdruck einer organischen, psychischen oder funktionellen Erkrankung. Trotz der Fülle der Differenzialdiagnose gelingt es meist durch sorgfältige Anamnese und wenige Untersuchungen, den Auslöser zu finden. Die Ursachen akuter Beschwerden unterscheiden sich teilweise von denen chronischer. Bei Frauen im gebärfähigen Alter ist eine Schwangerschaft auszuschließen. Eine symptomatische Therapie kann bereits zu Beginn eingeleitet werden. Die Klärung chronischer Ursachen ist schwieriger. Funktionstests wie die Messung der Magenentleerung ergänzen die Diagnostik. Sie sollen nur in spezialisierten Zentren mit entsprechender Erfahrung durchgeführt werden. Ruminieren und Regurgitation sind von Erbrechen abzugrenzen. Findet sich keine Ursache für die Beschwerden, kann von einer funktionellen Erkrankung ausgegangen werden
\end{abstract}

DOI: https://doi.org/10.1007/s11377-007-0084-7

Posted at the Zurich Open Repository and Archive, University of Zurich

ZORA URL: https://doi.org/10.5167/uzh-156705

Journal Article

Published Version

Originally published at:

Janiak, P; Fried, M (2007). Differenzialdiagnose Nausea und Erbrechen. Der Gastroenterologe, 2(3):201211.

DOI: https://doi.org/10.1007/s11377-007-0084-7 
Gastroenterologe 2007 $\cdot 2: 201-211$

DOI 10.1007/s11377-007-0084-7

Online publiziert: 11. April 2007

๑) Springer Medizin Verlag 2007

\section{Rubrikherausgeber}

G. Gerken, Essen

R. Schmid, München

H.J. Schulz, Berlin

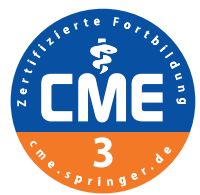

CME.springer.de - Zertifizierte Fortbildung für Kliniker und niedergelassene Ärzte

Die CME-Teilnahme an diesem Fortbildungsbeitrag erfolgt online auf CME.springer.de und ist Bestandteil des Individualabonnements dieser Zeitschrift. Abonnenten können somit ohne zusätzliche Kosten teilnehmen.

Unabhängig von einem Zeitschriftenabonnement ermöglichen Ihnen CME.Tickets die Teilnahme an allen CME-Beiträgen auf CME.springer.de. Weitere Informationen zu CME.Tickets finden Sie auf CME.springer.de.

\section{Registrierung/Anmeldung}

Haben Sie sich bereits mit Ihrer Abonnementnummer bei CME.springer.de registriert? Dann genügt zur Anmeldung und Teilnahme die Angabe Ihrer persönlichen Zugangsdaten. Zur erstmaligen Registrierung folgen Sie bitte den Hinweisen auf CME.springer.de.

\section{Online teilnehmen}

\section{und 3 CME-Punkte sammeln}

Die CME-Teilnahme ist nur online möglich. Nach erfolgreicher Beantwortung von mindestens 7 der $10 \mathrm{CME}$-Fragen senden wir Ihnen umgehend eine Bestätigung der Teilnahme und der $3 \mathrm{CME}$ Punkte per E-Mail zu.

\section{Zertifizierte Qualität}

Diese Fortbildungseinheit ist zertifiziert von der Landesärztekammer Hessen und der Nordrheinischen Akademie für Ärztliche Fort- und Weiterbildung und damit auch für andere Ärztekammern anerkennungsfähig.

Folgende Maßnahmen dienen der Qualitätssicherung aller Fortbildungseinheiten auf CME.springer.de: Langfristige Themenplanung durch erfahrene Herausgeber, renommierte Autoren, unabhängiger Begutachtungsprozess, Erstellung der CME-Fragen nach Empfehlung des IMPP mit Vorabtestung durch ein ausgewähltes Board von Fachärzten.

Für Fragen und Anmerkungen stehen wir Ihnen jederzeit zur Verfügung:

Springer Medizin Verlag GmbH

Fachzeitschriften Medizin/Psychologie

CME-Helpdesk, Tiergartenstraße 17

69121 Heidelberg

E-Mail: cme@springer.com

CME.springer.de

\author{
P. Janiak · M. Fried \\ Klinik für Gastroenterologie und Hepatologie, Universitätsspital Zürich
}

\section{Differenzialdiagnose Nausea und Erbrechen}

\section{Zusammenfassung}

Nausea und Erbrechen sind häufig. Sie können eine physiologische Antwort auf ein exogenes Toxin sein, aber auch Ausdruck einer organischen, psychischen oder funktionellen Erkrankung. Trotz der Fülle der Differenzialdiagnose gelingt es meist durch sorgfältige Anamnese und wenige Untersuchungen, den Auslöser zu finden. Die Ursachen akuter Beschwerden unterscheiden sich teilweise von denen chronischer. Bei Frauen im gebärfähigen Alter ist eine Schwangerschaft auszuschließen. Eine symptomatische Therapie kann bereits zu Beginn eingeleitet werden. Die Klärung chronischer Ursachen ist schwieriger. Funktionstests wie die Messung der Magenentleerung ergänzen die Diagnostik. Sie sollen nur in spezialisierten Zentren mit entsprechender Erfahrung durchgeführt werden. Ruminieren und Regurgitation sind von Erbrechen abzugrenzen. Findet sich keine Ursache für die Beschwerden, kann von einer funktionellen Erkrankung ausgegangen werden.

\section{Schlüsselwörter}

Nausea $\cdot$ Erbrechen $\cdot$ Emetogene Pharmaka $\cdot$ Regurgitation $\cdot$ Rumination

\section{Differential diagnosis: nausea and vomiting}

\begin{abstract}
Nausea and vomiting are common. They can be a physiological response to an exogenous toxin or indicate a organic, psychological or functional disease. Despite the variety of possible causes, careful attention to the patient's history and a few simple investigations usually make a diagnosis possible. Acute nausea and vomiting have causes that are differentin some ways from those of chronic nausea and vomiting. In women of child-bearing age pregnancy should be excluded. Symptomatic therapy can be initiated at once. Tracing chronic causes often presents a greater clinical challenge. Functional tests, such as those assessing gastric emptying, are applied if necessary. Vomiting must be distinguished from regurgitation and rumination. In patients whose symptoms remain unexplained we have to consider functional nausea and vomiting.
\end{abstract}

\section{Keywords}

Nausea $\cdot$ Vomiting $\cdot$ Emetic agents $\cdot$ Regurgitation $\cdot$ Rumination 
Die physiologische Funktion des Erbrechens besteht in der Entfernung von Toxinen

\section{- Brechzentrum}

Cannabinoide Rezeptoren führen im Brechzentrum zu einer Hemmung
Nausea und Erbrechen sind häufige Beschwerden, die durch eine Vielzahl von Ursachen und Erkrankungen ausgelöst werden können. Sie können Ausdruck einer organischen oder psychischen Erkrankung sein, aber auch die physiologische Antwort auf ein exogenes Toxin. Beide Symptome können gemeinsam oder getrennt auftreten; wenn sie gemeinsam auftreten, dann oft als Manifestation eines physiologischen Reflexes ausgelöst durch das Brechzentrum in der Medulla oblongata. Während Erbrechen ein komplexer, neurologischer Vorgang ist, kann Nausea auch ohne Aktivierung des Brechzentrums auftreten. Die Differenzialdiagnose umfasst neben medikamentös-toxischen, infektiösen und endokrinologischen Ursachen, Erkrankungen des Darmes, des ZNS auch funktionelle Ursachen. Wichtig ist die Unterscheidung zwischen akuten und chronischen Beschwerden. Trotz der breiten Differenzialdiagnose kann die Ursache durch eine gezielte Anamnese, den klinischen Kontext, eine körperliche Untersuchung und einfache diagnostische Untersuchungen meist schnell gefunden werden. Eine symptomatische Therapie kann von Anfang an erfolgen. Der Artikel gibt eine Übersicht über die wichtigsten Differenzialdiagnosen und deren Abklärung.

Die Prävalenz von Nausea und Erbrechen liegt in der Allgemeinbevölkerung bei 2-8\% pro Monat. Der Begriff Nausea ist vom griechischen Wort „naos“, Schiff, abgeleitet, wahrscheinlich, da Nausea häufig auf Schiffen beobachtet wurde. Er bezeichnet eine unangenehme Empfindung im Bereich des Epigastriums oder der Kehle mit dem Bedürfnis zu erbrechen. Erbrechen ist ein komplexer physiologischer Vorgang, der zum kräftigen Ausstoßen von Mageninhalt in den Mund und nach außen führt. Der Vorgang ist assoziiert mit Kontraktionen der Abdomen- und Thoraxmuskulatur, die zu einer Steigerung des intraabdominalen Druckes führen. Die physiologische Funktion des Erbrechens besteht in der Entfernung von Toxinen aus dem Darm und beinhaltet sowohl willkürliche als auch unwillkürliche Komponenten. Die neurologische Koordination geschieht im - Brechzentrum der Medulla oblongata, im dorsalen Bereich der lateralen Formatio reticularis. Afferente Bahnen kommen aus verschiedenen Teilen des Verdauungsapparates wie Pharynx, Magen oder Dünndarm, aber auch aus dem Herzen und den Hoden. Das Brechzentrum wird auch durch die Triggerzone im Bereich der Area postrema am Boden des vierten Ventrikels aktiviert. Die Triggerzone reagiert auf endogene und exogene Moleküle, die Erbrechen auslösen können. Schließlich existieren afferente Bahnen aus dem Cortex cerebri, dem Hirnstamm und dem Vestibularorgan [1]. Der Brechreflex wird durch verschiedene Rezeptoren moduliert. Die Stimulation von $5-\mathrm{HT}_{3}$-Serotonin-Rezeptoren führt zur Freisetzung von Dopamin, welches direkt Dopamin-2-Rezeptoren im Brechzentrum aktiviert. Dadurch erklärt sich die pharmakologische Wirkung von $5-\mathrm{HT}_{3}$-Inhibitoren wie Ondansetron und Dopaminrezeptor-Antagonisten wie Metoclopramid. Im Vestibularorgan finden sich Histamin- und Muskarinrezeptoren. Die Reiseerkrankung und Erkrankungen des Vestibularapparates sprechen deshalb auf H1-Rezeptorblocker und Anticholinergika an. Schließlich finden sich auch cannabinoide Rezeptoren, die im Brechzentrum zu einer Hemmung führen.

Vom Erbrechen müssen Regurgitation und Rumination unterschieden werden (• Tab. 1). Regurgitation bezeichnet das passive Zurückfließen von Mageninhalt in den Mund ohne Kontraktionen der Bauchwand- oder Zwerchfellmuskulatur [2]. Beim Ruminieren wird der regurgitierte Mageninhalt wiedergekäut und danach entweder erneut geschluckt oder herausgespuckt [2]. Rumination ist nicht begleitet von Nausea und beginnt meist wenige Minuten nach dem Essen. Es kann als Begleiterscheinung bei psychiatrischen Erkrankungen auftreten oder auch isoliert.

\section{Tab. 1 Definitionen}

\begin{tabular}{|ll|}
\hline Nausea & $\begin{array}{l}\text { Nausea bezeichnet eine unangenehme Empfindung im Bereich des Epigastriums oder der Kehle } \\
\text { mit dem Bedürfnis zu erbrechen. Nausea kann isoliert auftreten oder zusammen mit Erbrechen }\end{array}$ \\
Erbrechen & $\begin{array}{l}\text { Kräftiges Ausstoßen des Mageninhaltes in den Mund durch Kontraktionen der Bauchwand- und } \\
\text { Zwerchfellmuskulatur }\end{array}$ \\
\hline Regurgitation & $\begin{array}{l}\text { Passives Zurückfließen von Nahrung aus dem Magen zurück in den Mund ohne Kontraktion der } \\
\text { Bauchwand- oder Zwerchfellmuskulatur }\end{array}$ \\
\hline Rumination & $\begin{array}{l}\text { Kauen und Schlucken regurgitierter Nahrung, die durch eine willentliche Erhöhung des intra- } \\
\text { abdominalen Druckes zurück in den Mund befördert wurde. Rumination ereignet sich während } \\
\text { oder kurz nach dem Essen }\end{array}$ \\
\hline
\end{tabular}




\begin{tabular}{|c|c|c|c|}
\hline Infektiös & $\begin{array}{l}\text { Endokrine } \\
\text { und metabolische }\end{array}$ & Abdominal & ZNS \\
\hline Akute Gastroenteritis & Schwangerschaft & Mechanische Obstruktion & Migräne \\
\hline - Viral & Diabetes mellitus & Magenausgangsstenose & Neoplasien \\
\hline - Bakteriell & Ketoazidose & Stenose Dünndarm & Blutungen \\
\hline \multirow[t]{17}{*}{ Nichtgastrointestinale Infekte } & Hyperparathyreoidismus & Fremdkörper & Infarkte \\
\hline & Hyperkalzämie & Motilitätsstörung & Abszess \\
\hline & Hypoparathyreoidismus & lleus & Hydrocephalus \\
\hline & Hyponatriämie & Gastroparese & - Hoher Druck \\
\hline & Hyperthyreose & Chronische intestinale Pseudoobstruktion & - Tiefer Druck \\
\hline & M. Addison & Entzündliche extraintestinale Ursachen & - Kongenitale Malformationen \\
\hline & $\begin{array}{l}\text { Akute intermittierende } \\
\text { Porphyrie }\end{array}$ & Ulcus ventriculi und duodeni & Epilepsien \\
\hline & & Pankreatitis & Meningitis \\
\hline & & Akute Hepatitis & Autonome Erkrankungen \\
\hline & & Akute Cholezystitis & Demyelinisierende Erkrankungen \\
\hline & & Akute Appendizitis & Erkrankungen des Labyrinths \\
\hline & & M. Crohn & - Labyrinthitis \\
\hline & & Neoplasien & - Reiseerkrankung \\
\hline & & Magen, Pankreas u. a.karzinom & - M. Menière \\
\hline & & Metastasen & - Otitis media \\
\hline & & Mesenterialinfarkt & Viszerale Neuropathien \\
\hline & & Mesenteriale und retroperitoneale Prozesse & \\
\hline
\end{tabular}

\section{Spezifische Ursachen}

Neben physiologischen Ursachen (z. B. Schwangerschaft) sind Erkrankungen des Gastrointestinaltraktes, anderer abdominaler Organe und des ZNS häufig. Daneben gibt es toxische, metabolische und endokrine Ursachen (• Tab. 2, 3, 4). Trotz der zahlreichen Differenzialdiagnosen kann die Ursache meist gefunden und eine spezifische Therapie eingeleitet werden. Die Dauer der Beschwerden gibt bereits einen wichtigen Hinweis, da sich die Ursachen akuter und chronischer Beschwerden teilweise unterscheiden.

\section{Akute Ursachen}

\section{Medikamente und toxische Ursachen}

Nausea und Erbrechen als Nebenwirkungen von Medikamenten sind häufig. Meist tritt eine Nausea früh im Verlauf auf. Der zeitliche Zusammenhang ist meist wegweisend. Zahlreiche Medikamentenklassen sind als Auslöser bekannt, einige sind jedoch besonders häufig (• Tab. 3). Bei einigen Medikamenten ist der Wirkungsmechanismus bekannt. Zu den häufigsten Ursachen gehören die nichtsteroidalen Antirheumatika, wahrscheinlich durch Aktivierung von vagalen Afferenzen, die das Brechzentrum in der Medulla aktivieren. Andere Substanzen wie Nikotin, L-Dopa und Opiate wirken direkt auf die Triggerzone in der Area postrema. Häufig werden Nausea und Erbrechen auch bei Einnahme von Antibiotika wie Mebendazol, Digitalis, oralen Blutzuckersenkern und Azathioprin beschrieben.

Am besten untersucht sind Nausea und Erbrechen durch Chemotherapie. Unterschieden wird eine akute Nausea (innerhalb $24 \mathrm{~h}$ ), eine verspätete (nach $24 \mathrm{~h}$ ) und eine antizipatorische Nausea [3]. Risikofaktoren für ein akutes Erbrechen sind eine fehlende antiemetische Therapie, stark emetogene Substanzen, Nausea vor Beginn der Chemotherapie, weibliches Geschlecht und niedriger sozioökonomischer Status [4]. Das emetogene Potenzial des Chemotherapeutikums und die Wirksamkeit der antiemetischen Therapie gelten als Hauptrisikofaktoren [5]. Für die Prophylaxe und Therapie des akuten Erbrechens sind 5- $\mathrm{HT}_{3}$-Rezeptor-Antagonisten wie Ondansetron oder Tropisetron unerlässlich. Heute steht zusätzlich der Neurokinin-1-Rezeptor-Antagonist Apretiant zur Verfügung. Bei Verwendung hoch emetogener Substanzen wird eine Kombination aus 5- $\mathrm{HT}_{3}$-Rezeptor-Antagonisten mit einem NK-1-Rezeptor-Antagonist und Steroiden empfohlen [6, 7]. Der Hauptrisikofaktor
Die Dauer der Beschwerden gibt bereits einen wichtigen Hinweis

Der zeitliche Zusammenhang ist meist wegweisend

Nichtsteroidale Antirheumatika

Bei Verwendung hoch emetogener Substanzen wird eine Kombination aus $5-\mathrm{HT}_{3}$-Rezeptor-Antagonist, NK1-Rezeptor-Antagonist und Steroiden empfohlen 


\section{- Antizipatorisches Erbrechen}

Auslösend für die Nausea sind Schädigungen der Mukosa und auch eine direkte Wirkung von Toxinen auf die Triggerzone

Die Reiseerkrankung führt zu einer chronischen Aktivierung des Vestibularapparates und des Brechzentrums

- Emotionale Reaktionen

Tab. 3 Medikamentöse und toxische Ursachen. (Mod. nach [2])

\section{Substanzklasse}

Chemotherapeutika $^{\mathrm{a}}$

\begin{tabular}{l} 
\\
\hline Nichtsteroidale Antirheumatika $^{\mathrm{a}}$ \\
Opiate $^{\mathrm{a}}$ \\
\hline Antihypertensiva $^{\mathrm{a}}$
\end{tabular}

Antihypertensiva

Antiarrhythmika

Antibiotika $^{\mathrm{a}}$

\begin{tabular}{|c|c|}
\hline & Mebendazol \\
\hline & Tetrazykline \\
\hline & Antituberkulostatika \\
\hline & Sulfonamide \\
\hline & Aciclovir \\
\hline \multicolumn{2}{|l|}{ Orale Kontrazeptiva ${ }^{a}$} \\
\hline \multicolumn{2}{|l|}{ Orale Antidiabetika ${ }^{a}$} \\
\hline \multirow[t]{3}{*}{ Zentral wirksame Substanzen } & $\begin{array}{l}\text { Antiparkinson-Medikamente (z. B. } \\
\text { L-Dopa) }\end{array}$ \\
\hline & Antiepileptika \\
\hline & Nikotin (auch Pflaster) \\
\hline Antiasthmatika & Theophyllin \\
\hline \multirow[t]{2}{*}{ Toxische Ursachen } & Vitamin-A-Überdosierung \\
\hline & Bestrahlung \\
\hline
\end{tabular}

bensjahr. Virale Gastroenteritiden werden durch Rota-, Calci-, Reo- und Adenoviren ausgelöst, bakterielle Infektionen durch Staphylococcus aureus, Salmonellen, Bacillus cereus und Clostridium perfringens. Auslösend für die Nausea sind Schädigungen der Mukosa und auch eine direkte Wirkung von Toxinen auf die Triggerzone. Auch nicht gastrointestinale Infektionen wie eine Meningitis, Otitis media oder Hepatitis können zu Beschwerden führen.

\section{Neurologische Ursachen}

Jede Erkrankung des ZNS oder der Meningen kann Nausea und Erbrechen auslösen. Eine Erhöhung des intrakraniellen Druckes durch einen Tumor, Blutung, Infektion oder eine kongenitale Missbildung kann durch Aktivierung der Brechzentrums zu Erbrechen führen. Migraine Attacken, mit oder ohne Kopfschmerzen, gehören zu den häufigeren Ursachen.

Die Reiseerkrankung führt zu einer chronischen Aktivierung des Vestibularapparates und des Brechzentrums. Da die Aktivierung v. a. über Histamin- und Cholinrezeptoren erfolgt, sind Antihistaminika und Anticholinergika am besten wirksam. Andere häufige Ursachen sind eine virale Labyrinthitis sowie der M. Menière.

Psychiatrische Erkrankungen wie Angststörungen, Depressionen oder eine Anorexia nervosa sind mit Nausea und Erbrechen assoziiert. Daneben können - emotionale Reaktionen auf unangenehme Gerüche oder andere Sinneswahrnehmungen bzw. die bloße Erinnerung daran Nausea auslösen.

\section{Mechanische Obstruktion}

Eine peptische Stenose des Magenausganges war vor 1980 in 12\% der Fälle die Erstmanifestation eines Magenulkus. Heute ist die Magenausgangsstenose eine Rarität; sie kann sich abrupt oder allmählich manifestieren. Seltene Ursachen einer akuten Obstruktion mit Nausea und Erbrechen sind ein Magenvolvulus oder eine paraösophageale Hernie. Eine intestinale Obstruktion kann durch Inkarzeration einer intestinalen Hernie oder einen Tumor verursacht werden. Als Ursache einer neoplastischen 
Obstruktion kommen Metastasen, Dünndarmkarzinome, Lymphome oder Karzinoide in Frage.

\section{Postoperative Nausea und Erbrechen}

Postoperative Nausea und Erbrechen sind mehr ein therapeutisches als ein diagnostisches Problem. Differenzialdiagnostisch abzugrenzen sind chirurgische und kardiovaskuläre Komplikationen. Die Prävalenz zeigt eine hohe Variabilität. In einer Studie mit fast 4000 Patienten klagten 37\% der Patienten

\begin{tabular}{|c|c|}
\hline \multicolumn{2}{|l|}{ Postoperative Phase } \\
\hline \multicolumn{2}{|l|}{ Alkoholabusus } \\
\hline \multirow[t]{2}{*}{ Kollagenosen } & Systemische Sklerodermie \\
\hline & Lupus erythematodes \\
\hline \multicolumn{2}{|l|}{ Nach Vagotomie } \\
\hline \multirow[t]{3}{*}{ Psychiatrische Störungen } & Depressionen \\
\hline & Angsterkrankungen \\
\hline & Essstörungen \\
\hline \multirow[t]{2}{*}{ Internistische Erkrankungen } & Myokardinfarkt \\
\hline & Nephrolithiasis \\
\hline \multicolumn{2}{|l|}{ Intensive Schmerzen } \\
\hline \multicolumn{2}{|l|}{ Emotionaler Stress } \\
\hline \multirow[t]{3}{*}{ Funktionelle Erkankungen } & Chronische Nausea und Erbrechen \\
\hline & Funktionelles Erbrechen \\
\hline & „cyclic vomiting syndrome" \\
\hline
\end{tabular}
nach Vollnarkose über Nausea, 23\% der Patienten mussten erbrechen [10]. Das Risiko ist von verschienen Faktoren abhängig. Frauen haben ein 3 fach höhere Risiko. Eine Vollnarkose, inhalative Anästhetika und lange dauernde Operation sind ebenfalls mit einem höherem Risiko verbunden [11]. Postoperative Nausea und Erbrechen sind nach gynäkologischen, abdominalen und orthopädischen Operationen häufiger [12].

\section{Intestinale Ischämie}

Eine intestinale Ischämie kann sich mit $\triangleright$ akutem Erbrechen äußern. Je nach zugrundeliegendem Mechanismus kann die klinische Präsentation von akuten Schmerzen und Erbrechen bis zu unspezifischen Beschwerden, die im Verlauf zunehmen, reichen. Vorhofflimmern, Alter $>60$, Herzinsuffizienz, frischer Myokardinfarkt, postprandiale Bauchschmerzen und Gewichtsverlust lassen insbesondere an ein vaskuläres Ereignis denken [13]. Bei entsprechendem Verdacht ist eine frühzeitige Abklärung wichtig.

\section{Extraintestinale Ursachen}

Extraintestinale Ursachen stellen meist keine diagnostischen Probleme dar, da ihr Ursprung in der Regel klinisch zuzuordnen ist. Jeder abdominale Schmerz kann Auslöser von Nausea und Erbrechen sein. Neben entzündlichen Veränderungen des Peritoneums (z. B. durch eine Darmperforation oder akute Appendizitis) sind renale und biliäre Koliken stark emetogen. Das fulminante Leberversagen aber auch die Chemoembolisation von hepatozellulären Karzinomen können für Beschwerden verantwortlich sein [14]. Ein Myokardinfarkt kann sich als Nausea manifestieren, wobei die Beschwerden eher von der Infarktgröße als von der Lokalisation abhängig sind [15]. Schließlich sind auch die Torsion von Hoden und Ovar mit intensiver Nausea und Erbrechen assoziiert.

\section{Endokrinologische und metabolische Ursachen}

Die Differenzialdiagnose umfasst metabolische Ursachen wie Urämie, Ketoazidose, Hyponatriämie und Hyperkalzämie, daneben endokrinologische Störungen wie Hyperthyreose, Hyper- und Hypoparathyreoidismus sowie die Addison-Erkrankung. Die Entstehung der Symptome ist noch unklar. Bei der Urämie und diabetischen Ketoazidose wird eine direkte $>$ Aktivierung der Area postrema angenommen.

\section{Schwangerschaft}

Nausea tritt bei 70-85\% aller Schwangerschaften auf [16]: 50\% der Frauen haben Nausea und Erbrechen, 25\% nur Nausea und 25\% keine Beschwerden [17, 18]. Die Symptome beginnen meist früh in der Schwangerschaft, erreichen um die 9. Schwangerschaftswoche ihr Maximum und dauern selten länger als 22 Wochen. Die Ursache für die Beschwerden ist unklar. Neben Hormonen wie $\beta-H C G$ oder Östrogenen sind auch psychologische Faktoren beschrieben. Die Beschwerden können jedoch bereits auftreten, bevor die Schwangerschaft bekannt ist. Deshalb muss bei jeder Frau im gebärfähigen Alter zunächst ein Schwangerschaftstest durchgeführt werden. Risikofaktoren sind multiple
Auch eine Vollnarkose, inhalative Anästhetika und lange dauernde Operation sind mit einem höherem Risiko verbunden

\section{- Akutes Erbrechen}

Jeder abdominale Schmerz kann Auslöser von Nausea und Erbrechen sein

\section{Aktivierung der Area postrema}

Bei jeder Frau im gebärfähigen Alter muss zunächst ein Schwangerschaftstest durchgeführt werden 
Nicht immer lässt sich eine spezifische Ursache für die verzögerte Magenentleerung identifizieren

Zur Messung einer verzögerten Magenentleerung werden die Szintigraphie und Atemtests mit Messung stabiler, nicht radioaktiver Isotopen $\left({ }^{13} \mathrm{C}\right.$ Octanoat) verwendet

- Rome-III-Kriterien
Schwangerschaften, eine Hyperemesis gravidarum in einer vorangegangen Schwangerschaft, sowie eine positive Familienanamnese [19].

\section{Chronische Ursachen}

Die Differenzialdiagnose bei chronischen Beschwerden wird erweitert durch Nausea und Erbrechen in der Schwangerschaft, bei Gastroparese, wegen Motilitätsstörungen im Rahmen von neurologischen Erkrankungen sowie funktionelle Störungen.

\section{Gastroparese und Motilitätsstörungen des Dünndarms}

Gastroparese wird definiert als chronische Störung der Magenentleerung mit entsprechenden Symptomen ohne mechanische Obstruktion. Die Symptome einer Gastroparese wie frühes Sättigungsgefühl, Nausea, Erbrechen und epigastrische Beschwerden sind sehr variabel und korrelieren nicht immer mit der gemessenen Magenentleerungsstörung [20]. Bei Patienten mit Diabetes mellitus liegt die Prävalenz von Symptomen einer gastrointestinalen Motilitätsstörung bei 11-18\% [21, 22]. In einer szintigraphischen Studie hingegen zeigte sich bei 65\% der Patienten mit Diabetes mellitus eine verzögerte Magenentleerung [23]. Eine spezifische Ursache für die verzögerte Magenentleerung lässt sich nicht immer identifizieren. Etwa 25-30\% der Patienten haben einen seit langer Zeit bestehenden Diabetes mellitus, in über 30\% ist die Ursache unklar, idiopathisch. Weitere Ursachen sind u. a. Zustand nach Magenoperation bzw. Vagotomie, M. Parkinson, Kollagenosen sowie die chronisch intestinale Pseudoobstruktion [24, 25]. Die Diagnose einer Gastroparese beruht auf den typischen Symptomen und dem Nachweis einer verzögerten Magenentleerung. Eine mechanische Obstruktion muss vorher ausgeschlossen werden. Typische Beschwerden allein reichen nicht aus, da eine rasche Magenentleerung zu ähnlichen Symptomen führen kann.

Zur Messung einer verzögerten Magenentleerung werden die Szintigraphie und Atemtests mit Messung stabiler, nicht radioaktiver Isotopen $\left({ }^{13} \mathrm{C}\right.$-Octanoat $)$ verwendet [26, 27]. Die Elektrogastrographie und die Sonographie haben in der klinischen Diagnostik bisher keinen Platz.

Eine verzögerte Magenentleerung wurde auch bei Patienten mit einer funktionellen Dyspepsie beobachtet, unabhängig von der Art der Dyspepsie. Die Bedeutung der Magenentleerungsstörung für die Symptombildung ist hier noch unklar.

\section{Helicobacter-pylori-Infektion}

Eine Infektion mit Helicobacter pylori findet sich bei etwa $80 \%$ der Patienten mit einem Ulcus duodeni und bei etwa 60\% mit einem Ulcus ventriculi, bei einer funktionellen Dyspepsie nur bei 20-30\%. Eine Helicobacter-Infektion zeigt nur eine (geringe) Assoziation mit dyspeptischen Beschwerde, eine Eradikation führte nur bei $9 \%$ der Patienten zu einer Verbesserung der dyspeptischen Symptome $[28,29]$. Eine Helicobacter-pylori-Gastritis ist keine Erklärung für Nausea und Erbrechen.

\section{Partielle intestinale Obstruktion}

Im Unterschied zur kompletten intestinalen Obstruktion sind die Symptome chronisch und rezidivieren über lange Zeit. Die Differenzialdiagnose umfasst Stenosen bei M. Crohn, Neoplasien, eine Peritonealkarzinose oder ischämische Strikturen. Adhäsionen nach Operationen oder im Rahmen von entzündlichen Prozessen können Beschwerden verursachen, der kausale Zusammenhang ist jedoch oft fraglich.

\section{Neurologische Erkrankungen}

$\mathrm{Zu}$ den neurologischen Erkrankungen mit chronischer Nausea und Erbrechen gehören die Migräne und der Hydrocephalus.

\section{Funktionelle Nausea und Erbrechen}

Chronische Beschwerden ohne eindeutige Ursachen werden als funktionelle Störung bezeichnet. Epidemiologische Studien zeigen, dass gelegentliches Erbrechen bei etwa 2-3\% der Allgemeinbevölkerung vorkommt, die wenigsten dieser Personen erfüllen jedoch die Kriterien für eine funktionelle Störung. Die funktionellen Störungen mit Nausea und Erbrechen werden gemäß den neuen $>$ Rome-IIIKriterien in drei Gruppen unterteilt: chronisch idiopathische Nausea, funktionelles Erbrechen und das „cyclic vomiting syndrome“ [30]. Die diagnostischen Kriterien müssen in den letzten 3 Monaten 
erfüllt worden sein und der Beginn der Symptome sollte länger als 6 Monate zurückliegen. Patienten mit einer offensichtlichen psychischen Erkrankung gehören nicht in diese Gruppe.

Die chronisch idiopathische Nausea wird als störende Nausea an mehreren Tagen der Woche definiert. Sie tritt meist ohne Erbrechen auf.

Chronisches Erbrechen mindestens einmal pro Woche ohne Hinweis auf eine manifeste psychiatrische Erkrankung wird als funktionelles Erbrechen definiert. Das Erbrechen darf nicht willentlich provoziert sein. Strukturelle, metabolische und neurologische Ursachen müssen ausgeschlossen werden. Eine Essstörung oder ein Cannabis-Abusus dürfen ebenfalls nicht vorliegen. Die Ätiologie dieser Erkrankungen ist unklar, das Ansprechen auf trizyklische Antidepressiva spricht für eine psychische Komponente [31].

\section{Cyclic vomiting syndrome}

Das „cyclic vomiting syndrome“ ist charakterisiert durch ein periodisches Auftreten von Brechepisoden. Die Episoden können einen Tag bis mehrere Wochen andauern und haben meist einen stereotypen Ablauf. Zwischen den Episoden liegen asymptomatische Phasen, teilweise persistiert jedoch eine leichte Nausea. Einige Patienten berichten über migräneähnliche Prodromalsymptome vor den Episoden. Das mittlere Alter bei Auftreten der Beschwerden ist 5 Jahre. Obwohl selten kann das „cylcic vomiting syndrome“ auch bei Erwachsenen auftreten, die Assoziation mit Migräne ist in dieser Altersgruppe jedoch schwächer. Eine familiäre Belastung ist besonders bei Jugendlichen häufig. Bei Frauen wurde eine Assoziation mit dem Menstruationszyklus beschrieben.

\section{Ruminieren}

Ruminieren bezeichnet ein wiederholtes Regurgitieren von Speisen vom Magen in den Mund ohne ersichtliche Anstrengung. Die regurgitierte Nahrung wird anschließend gekaut und entweder erneut geschluckt oder herausgespuckt. Nausea und andere autonome Begleiterscheinungen, wie beim Erbrechen üblich, treten beim Ruminieren nicht auf. Das Ruminieren beginnt meist kurz nach Ende der Mahlzeit oder bereits während des Essens und dauert üblicherweise 1-2 Stunden [30]. Eingeleitet wird das Regurgitieren der Nahrung durch eine willkürliche Kontraktion des M. rectus abdominis. Unwillkürliche Kontraktionen der Bauchmuskulatur oder des Zwerchfells wie beim Erbrechen treten nicht auf. Gelegentlich kann die Erkrankung zu Symptomen einer Refluxerkrankung, zu Diarrhö oder Gewichtsverlust führen. Eine Assoziation mit Bulimie und anderen funktionellen Erkrankungen wurde beschrieben.

\section{Diagnostisches Vorgehen}

\section{Anamnese}

Bei der Breite der Differenzialdiagnose ist die Anamnese entscheidend für das weitere diagnostische Vorgehen (• Abb. 1). Die Anamnese wird durch wenige serologische Bestimmungen ergänzt. Bei Frauen im entsprechenden Alter gehört ein Schwangerschaftstest zur Basisdiagnostik.

Differenzialdiagnostisch stehen bei akuten Beschwerden ein Infekt, eine medikamentös-toxische oder eine metabolische Ursache im Vordergrund. Neben der Dauer der Beschwerden können Zeitpunkt und Begleitumstände wichtige Hinweise geben. Morgendliches Erbrechen tritt typischerweise in der Schwangerschaft, bei Urämie, Alkoholabusus und erhöhtem intrakraniellem Druck auf. Ein - postprandiales Intervall von einer Stunde spricht für eine Gastroparese oder eine Stenose, während bei psychischen Beschwerden das Erbrechen meist bereits während oder kurz nach dem Essen auftritt. Begleitsymptome wie Fieber, Diarrhö, Bauch- oder Kopfschmerzen geben oft Hinweise auf mögliche Auslöser. Bauchschmerzen deuten auf eine entzündliche Ursache (Appendizitis, Cholezystitis etc.) oder einen mechanischen bzw. paralytischen Ileus hin. Neurologische Symptome sind gezielt zu erfragen. Neben fokalen Symptomen ist auf eine Beeinträchtigung der Vigilanz zu achten. Schwindel und Tinnitus sprechen für eine Innenohrstörung. Das Regurgitieren unverdauter Nahrung lassen an eine Achalasie oder ein Zenker-Divertikel denken. Teilverdaute Nahrung wird bei einer Gastroparese oder einer Stenose des Magenausganges beobachtet. Fäkulentes Erbrechen tritt bei einer distalen Obstruktion, einem „bacterial overgrowth“ oder einer gastrokolischen Fistel auf.
Chronisch idiopathische Nausea

Strukturelle, metabolische und neurologische Ursachen müssen ausgeschlossen werden

Nausea und andere autonome Begleiterscheinungen, wie beim Erbrechen üblich, treten beim Ruminieren nicht auf

Unwillkürliche Kontraktionen der Bauchmuskulatur oder des Zwerchfells wie beim Erbrechen treten nicht auf

\section{- Postprandiales Intervall}

Begleitsymptome wie Fieber, Diarrhö, Bauch- oder Kopfschmerzen geben oft Hinweise auf mögliche Auslöser 


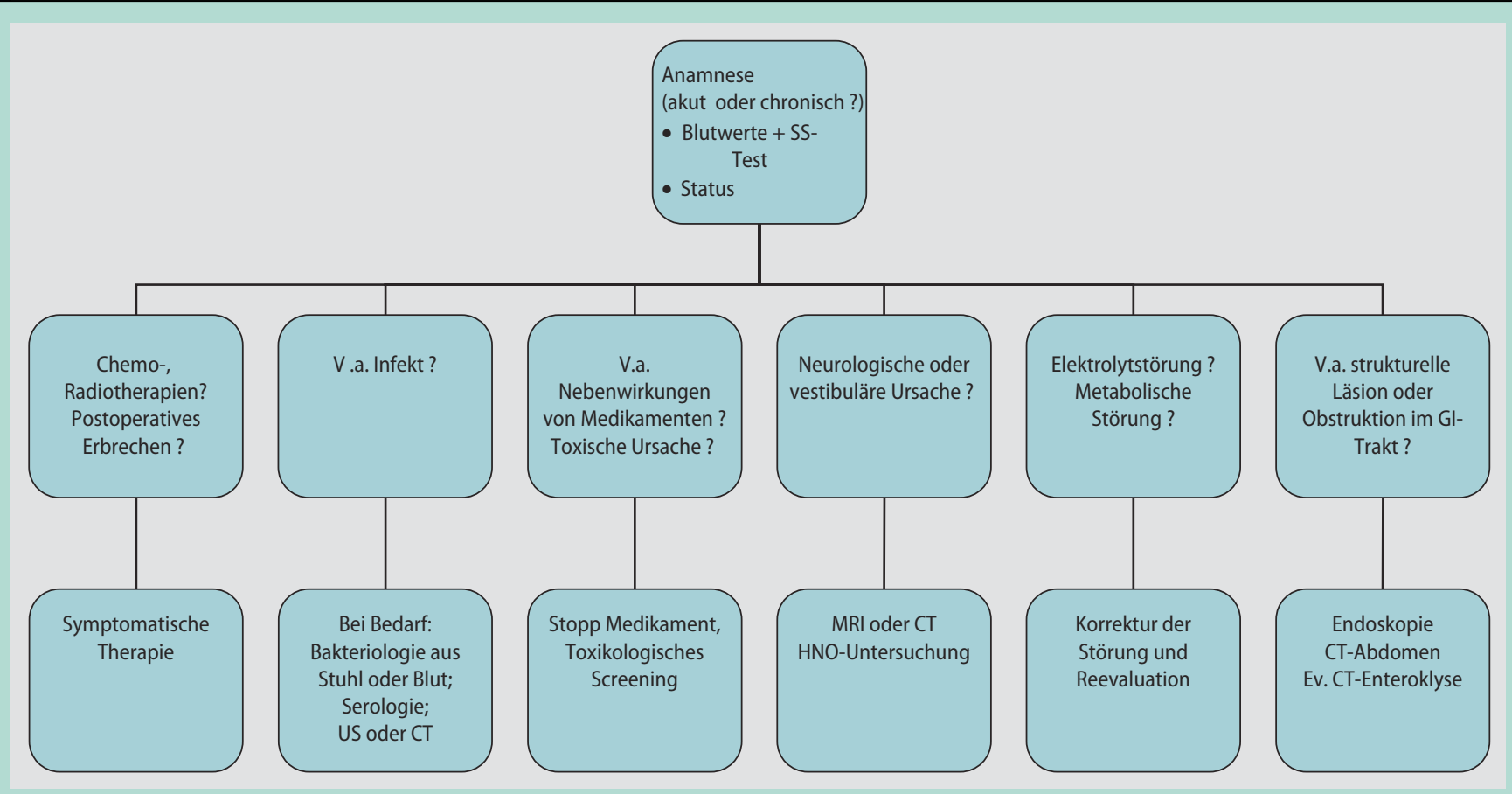

Abb. $1 \Delta$ Abklärung bei Nausea und Erbrechen

Beim akuten Erbrechen ist auf Anzeichen für eine Dehydratation zu achten und das Abdomen auf Druckdolenzen und Resistenzen zu untersuchen

\section{- Röntgenleeraufnahme des Abdomens}

\section{Eine Kapselendoskopie des Dünn- darms ist bei Verdacht auf eine Obstruktion kontraindiziert}

\section{${ }^{13}$ C-Atemtest}

\section{Körperliche Untersuchung}

Beim akuten Erbrechen ist auf Anzeichen für eine Dehydratation zu achten und das Abdomen auf Druckdolenzen und Resistenzen zu untersuchen. Die Auskultation der Darmgeräusche ist wichtig zur Beurteilung von Passagestörungen. Ikterische Skleren, vergrößerte Lymphknoten geben weitere diagnostische Hinweise. Die Untersuchung der Haut kann Hinweise auf eine Systemerkrankung wie eine Sklerodermie geben. Bei der neurologischen Untersuchung sind neben den Pyramidenbahnen v. a. die Hirnnerven zu untersuchen. Veränderungen der Blutdruck- und Pulsregulation werden bei Erkrankungen des autonomen Nervensystems beobachtet.

\section{Weitere Diagnostik}

Neben der Basisdiagnostik richten sich die weiteren Untersuchungen nach Anamnese und körperlichem Status. Die Einleitung einer symptomatischen Therapie kann bereits auf empirischer Basis erfolgen [2]. Bei Verdacht auf eine Passagestörung sollte eine $>$ Röntgenleeraufnahme des Abdomens durchgeführt werden. Bei einer partiellen Obstruktion können aber bei bis zu 22\% Zeichen eines Ileus fehlen [32]. Bei Verdacht auf eine Läsion im oberen Gastrointestinaltrakt ist primär eine Endoskopie indiziert, bei einer distalen Obstruktion eine CT-Untersuchung des Abdomens möglichst mit Dünndarmdarmstellung [33]. Zusätzlich können andere Ursachen wie eine intraabdominelle Raumforderung und pathologische Befunde im Bereich des Pankreas, Leber und Gallenwege erfasst werden. Eine Kapselendoskopie des Dünndarms ist bei Verdacht auf eine Obstruktion kontraindiziert.

Eine MRT des Schädels ist bei Verdacht auf eine zentrale Raumforderung dem CT überlegen, da Veränderungen des Hirnstammes bzw. des Kleinhirnbrückenwinkels besser zur Darstellung kommen.

Bei chronischem Erbrechen und Verdacht auf funktionelle Störung der Magenentleerung wird in der klinischen Diagnostik meist eine Magenentleerungsszintigraphie oder - soweit verfügbar ein - ${ }^{13}$ C-Atemtest verwendet. Die Elektrogastrographie und antroduodenale Manometrie steht nur in hochspezialisierten Zentren zur Verfügung. Beide Untersuchungen haben außerhalb von wissenschaftlichen Fragestellungen keine Bedeutung.

Trotz der breiten Differenzialdiagnose kann die Ursache für akute Nausea und Erbrechen in den meisten Fällen durch Anamnese und eine körperliche Untersuchung gefunden und eine gezielte Therapie eingeleitet werden. Mit einer symptomatischen Therapie kann sofort begonnen werden. Die Ur- 
sachen chronischer Beschwerden sind oft schwieriger zu erfassen; je nach Befund sind Funktionstests wie eine Untersuchung der Magenentleerung notwendig. Findet sich keine Ursache, müssen funktionelle Beschwerden in die Differenzialdiagnose einbezogen werden.

\section{Korrespondierender Autor Prof. M. Fried}

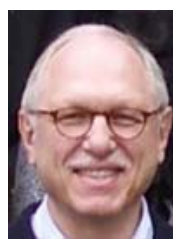

Klinik für Gastroenterologie und Hepatologie, Universitätsspital Zürich

Rämistrasse 100, 8091 Zürich

michael.fried@usz.ch

Interessenkonflikt. Es besteht kein Interessenkonflikt. Der korrespondierende Autor versichert, dass keine Verbindungen mit einer Firma, deren Produkt in dem Artikel genannt ist, oder einer Firma, die ein Konkurrenzprodukt vertreibt, bestehen. Die Präsentation des Themas ist unabhängig und die Darstellung der Inhalte produktneutral.

\section{Literatur}

1. Hornby PJ (2001) Central neurocircuitry associated with emesis. Am J Med 111: 106-112

2. Quigley E, Hasler W, Parkman $\mathrm{H}$ (2001) AGA technical review on nausea and vomiting. Gastroenterology 120: $263-286$

3. Jordan K, Voigt W, Schmoll HJ (2006) Chemotherapie-induziertes Erbrechen - Praktische Anleitung zur Prophylaxe und Therapie. Chemotherapy induced nausea and vomiting - a practical treatment approach. Dtsch Med Wochenschr: 1869-1872

4. Osoba D, Zee B, Pater J et al. (1997) Determinants of postchemotherapy nausea and vomiting in patients with cancer. Quality of Life and Symptom Control Committees of the National Cancer Institute of Canada Clinical Trials Group. J Clin Oncol 15: 116-123

5. Pater J, Slamet L, Zee B et al. (1994) Inconsistency of prognostic factors for post-chemotherapy nausea and vomiting. Supportive Care Cancer V2: 161-166

6. Kris MG, Hesketh PJ, Herrstedt J et al. (2005) Consensus proposals for the prevention of acute and delayed vomiting and nausea following highemetic-risk chemotherapy. Supportive Care Cancer V13: 85-96

7. Kris MG, Hesketh PJ, Somerfield MR et al. (2006) American Society of Clinical Oncology Guideline for Antiemetics in Oncology: Update 2006. J Clin Oncol 24: 2932-2947

8. Goedhals L, Heron J-F, Kleisbauer JP et al. (1998) Control of delayed nausea and vomiting with granisetron plus dexamethasone or dexamethasone alone in patients receiving highly emetogenic chemotherapy: A double-blind, placebo-controlled, comparative study. Ann Oncol 9: 661-666

9. Kacker V, Gupta Y (1996) An experimental model to study intracranial hypertension-induced vomiting in conscious dogs. Methods Find Exp Clin Pharmacol 18: 315
10. Quinn A, Brown J, Wallace P et al. (1994) Studies in postoperative sequelae. Nausea and vomiting - still a problem. Anaesthesia 49: 62-65

11. Kenny G (1994) Risk factors for postoperative nausea and vomiting. Anaesthesia 49: 6-10

12. Watcha M, White P (1993) Post operative nausea and vomiting; its etiology, treatment and prevention. Anaesthesiology 78: 403-408

13. Oldenburg WA, Lau LL, Rodenberg TJ et al. (2004) Acute mesenteric ischemia: A clinical review. Arch Intern Med 164: 1054-1062

14. Sohara N, Takagi H, Abe T et al. (1999) Nausea and vomiting induced by arterial chemo-embolization in patients with hepatocellular carcinoma and the antiemetic effect of ondansetron hydrochloride. Supportive Care Cancer V7: 84-88

15. Herlihy T, Mclvor ME, Cummings CC et al. (1987) Nausea and vomiting during acute myocardial infarction and its relation to infarct size and location. Am J Cardiol 60: 20-22

16. Jewell D, Young G (eds) (2003) Interventions for nausea and vomiting in early pregnancy (Cochrane Review). John Wiley, London

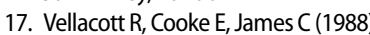
Nausea and vomiting in early pregnancy. Int J Gynaecol Obstet 27: 57-62

18. Gadsby R, Barnie-Adshead A, Jagger $C$ (1993) A prospective study of nausea and vomiting during pregnancy. Br J Gen Pract 43: 245-248; (Erratum in 43: 325)

19. ACOG Practice Bulletin \#52 (2004) Nausea and vomiting of pregnancy. Obstet Gynecol 103: 803

20. Horowitz M, Maddox A, Wishart JM et al. (1991) Relationships between oesophageal transit and solid and liquid gastric emptying in diabetes mellitus. Eur J Nucl Med 18: 229-234
21. Bytzer P, Talley NJ, Leemon $M$ et al. (2001) Prevalence of gastrointestinal symptoms associated with diabetes mellitus: A population-based survey of 15000 adults. Arch Intern Med 161: 1989-1996

22. Maleki D, Locke III GR, Camilleri M et al. (2000) Gastrointestinal tract symptoms among persons with $\mathrm{Di}$ abetes Mellitus in the community. Arch Intern Med 160: 2808-2816

23. Jones KL, Russo A, Stevens JE et al. (2001) Predictors of delayed gastric emptying in diabetes. Diabetes Care 24: $1264-1269$

24. Hasler W (2005) Nausea, gastroparesis, and aerophagia. J Clin Gastroenterol 39: s223-s228

25. Soykan I, Sivri B, Sarosiek I et al. (1998) Demography, clinical characteristics, psychological and abuse profiles, treatment, and long-term follow-up of patients with gastroparesis. Dig Dis Sci V43: 2398-2404

26. Tougas G, Eaker EY, Abell TL et al (2000) Assessment of gastric emptying using a low fat meal: establishment of international control values. Am J Gastroenterol 95: 1456-1462

27. Choi M, Camilleri M, Burton D et al. (1991) [13C]octanoic acid breath test for gastric emptying of solids: accuracy, reproducibility, and comparison with scintigraphy. Gastroenterology 112: 1155-1162

28. Jaakkimainen $R$, Boyle $E$, Tudiver $F$ (1999) Is Helicobacter pylori associated with non-ulcer dyspepsia and will eradication improve symptoms? A meta-analysis. BMJ 319: 1040 1044

29. Moayyedi P, Soo S, Deeks J et al. (2000) Systematic review and economic evaluation of Helicobacter pylori eradication treatment for non-ulcer dyspepsia. BMJ 321:659-664

30. Tack J, Talley NJ, Camilleri M et al. (2006) Functional gastroduodenal disorders. Gastroenterology 130: 1466-1479
31. Prakash C, Clouse RE (1999) Cyclic vomiting syndrome in adults: clinical features and response to tricyclic antidepressants. Am J Gastroenterol 94: 2855-2860

32. Shrake P, Rex D, Lappas J et al. (1991) Radiographic evaluation of suspected small bowel obstruction. Am J Gastroenterol 86: 175-178

33. Herlinger $H$ (1995) Guide to imaging of the small bowel. Gastroenterol Clin North Am 24: 309-329 
Bitte beachten Sie:

Antwortmöglichkeit nur online unter: CME.springer.de Die Frage-Antwort-Kombinationen werden online

\section{Welche Aussage zum Erbrechen} trifft zu?

$\square$ Das Brechzentrum liegt in der Area postrema.

$\square$ Es ist immer mit Nausea verbunden.

$\square$ Es ist assoziiert mit Kontraktionen der Zwerchfell- und Thoraxmuskulatur.

$\square$ Chronisches Erbrechen ist immer Zeichen eines fortgeschrittenen Karzinoms.

$\square$ Es spricht gegen eine funktionelle Erkrankung.

\section{Welche Aussage zur Gastro- parese trifft zu? \\ $\square$ Symptome wie Sättigungsge- fühl, Nausea und Erbrechen und geschluckt. \\ $\square$ Das Ruminieren kann bereits während der Mahlzeit begin- nen.} zeigen eine gute Korrelation mit der gemessenen Magenentleerungsstörung.

$\square$ Die beiden häufigsten Ursachen sind idiopathisch und diabetogen.

$\square$ Eine verzögerte Magenentleerung schließt eine funktionelle Dyspepsie aus.

$\square$ Sie ist bei M. Parkinson typischerweise nicht assoziiert.

$\square$ In der Diagnostik wird die Szintigraphie durch die Sonographie und Elektrogastrographie zunehmend verdrängt.
Welche Aussage zum Ruminieren trifft nicht zu?

$\square$ Ruminieren ist ein passiver

Vorgang ohne aktive Muskelkontraktion der Abdomenmuskulatur.

$\square$ Symptome wie saures Aufstoßen, Diarrhö oder ein Gewichtsverlust können vorhanden sein.

$\square$ Autonome Begleiterscheinungen wie Nausea treten üblicherweise nicht auf.

$\square$ Die regurgitierte Nahrung wird häufig wieder gekaut

Eine 34 jährige Frau meldet sich beim Hausarzt wegen persistierender Nausea und Erbrechen seit 6 Wochen. Vor einem Jahr wurde wegen dyspeptischen Beschwerden eine Gastroskopie durchgeführt, welche bis auf eine Helicobacterpylori-Gastritis unauffällig war. Eine Eradikation wurde bisher nicht durchgeführt. Welche Aussage ist falsch?

$\square$ Eine Helicobacter-pylori-Gastritis ist eine hinreichende Erklärung für die Beschwerden.

$\square$ Eine Helicobacter-pylori-Infektion findet sich in $20-30 \%$ der Patienten mit einer funktionellen Dyspepsie.

$\square$ Aufgrund des Alters und der Beschwerden sollte vor weiteren Abklärungen ein Schwangerschaftstest durchgeführt werden.
Nausea und Erbrechen über die 22. Schwangerschaftswoche hinaus sind selten.

$\square$ Eine Helicobacter Eradikation zur Therapie einer Nausea wird nicht empfohlen.

Das Brechzentrum wird durch verschiedene Rezeptoren moduliert. Welcher Rezeptor hat keine Bedeutung?

$\square$ 5-HT3-Rezeptor.

$\square$ Dopamin-Rezeptoren.

$\square$ H1-Rezeptor.

$\square$ Acetylcholin-Rezeptor.

$\square$ H2-Rezeptor.

Welche Aussage zu medikamentösen und toxischen Ursachen für Nausea und Erbrechen trifft nicht zu?

$\square$ Das Fehlen einer Ulkuserkrankung schließt nichtsteroidale Antirheumathika als Ursache von Nausea und Erbrechen aus.

$\square$ L-Dopa löst Nausea durch eine direkte Wirkung auf die Area postrema aus.

$\square$ Das emetogene Potential der Chemotherapie ist der Hauptrisikofaktor für akutes Erbrechen bei Chemotherapie.

$\square$ Bei hoch emetogenen Chemotherapien wird heute zur Prophylaxe von akutem Erbrechen eine Kombination aus Steroiden, 5-HT3-RezeptorAntagonisten und NK-1-Rezeptor-Antagonisten empfohlen.

$\square$ Chronischer Alkoholkonsum ist eine häufige unerkannte Ursache für chronische Nausea.
Welche Aussage zur Abklärung bei Nausea und Erbrechen trifft zu?

$\square$ Die Anamnese ist bei akuter Nausea und Erbrechen nur bedingt hilfreich.

$\square$ Das Fehlen radiologischer Zeichen für einen lleus in der Abdomen-Leeraufnahme schließt eine Obstruktion der Magendarmpassage aus.

$\square$ Zur Diagnose einer Gastroparese wird meist eine Szintigraphie zur Untersuchung der Magenentleerung oder ein 13C-Atemtest durchgeführt

$\square$ Mit einer symptomatischen Behandlung sollte bis zur Diagnosestellung zugewartet werden.

$\square$ Die Kapselendoskopie ist zur Abklärung einer chronischer Nausea indiziert. 
Welche Aussage zum „cyclic vomiting syndrome" und funktionellen Erbrechen ist falsch?

$\square$ Das "cyclic vomiting syndrome" tritt bei Erwachsenen häufiger auf als bei Jugendlichen und Kindern.

$\square$ Einige Patienten mit „,cyclic vomiting syndrome" berichten über migräneähnliche Symptome im Vorfeld.

$\square$ Bei Frauen mit "cyclic vomiting syndrome" wurde eine Assoziation mit dem Menstruationszyklus beschrieben.

$\square$ Bei funktionellem Erbrechen müssen Essstörungen und ein Cannabisabusus ausgeschlossen sein.

$\square$ Ein Ansprechen auf trizyklische Antidepressiva bei funktionellem Erbrechen ist in der Literatur beschrieben.

Welche Aussage zu infektiösen Ursachen ist richtig?

$\square$ Die höchste Prävalenz viraler Gastroenteritiden liegt zwischen dem 30. und 40. Lebensjahr.

$\square$ Nausea und Erbrechen beim Staphylococcus-aureus-Infekt werden durch eine direkte Toxinwirkung auf das Brechzentrum in der Medulla oblongata ausgelöst.

$\square$ Die häufigste Ursache einer viralen Gastroenteritis bei Kleinkindern sind Adenoviren.

$\square$ Die Reizung der Mukosa ist Auslöser der Nausea bei einer viralen Gastroenteritis

$\square$ Eine Reizung der Area postrema alleine reicht nicht aus, um Erbrechen auszulösen.
Eine 32-jährige Frau sucht die Notfallstation wegen Nausea, diffuser Abdominalschmerzen und Schwindel auf. Die Beschwerden haben vor 4 Tagen mit einer wässrigen Diarrhö begonnen. Die 3-jährige Tochter der Patientin leidet ebenfalls an einer Durchfallerkrankung. Die klinische Untersuchung zeigt ein weiches Abdomen mit einer diffusen Druckdolenz, die Darmgeräusche sind lebhaft. Axillär wird eine Temperatur von $37,7^{\circ} \mathrm{C}$ gemessen. Welche Aussage ist falsch?

$\square$ Ein Schwangerschaftstest sollte in dieser Situation durchgeführt werden.

$\square$ Eine Exsikkose sollte ausgeschlossen werden.

$\square$ Die Beschwerden sind durch eine virale Gastroenteritis bedingt, weitere Abklärungen sind unnötig

$\square$ Aufgrund der Anamnese ist eine Blutentnahme mit Bestimmung der Elektrolyte, des Kreatinins sowie des Blutzuckers angezeigt.

$\square$ Eine metabolische oder endokrinologische Ursache sollte in der Differenzialdiagnose berücksichtigt werden.

Diese Fortbildungseinheit ist

12 Monate auf CME.springer.de verfügbar. Den genauen Einsendeschluss erfahren Sie unter CME.springer.de 